Published in final edited form as:

Wiley Interdiscip Rev Nanomed Nanobiotechnol. 2013 July ; 5(4): 388-398. doi:10.1002/wnan.1223.

\title{
Glycosaminoglycans in Biomedicine
}

\author{
Rebecca A. Scott ${ }^{1}$ and Alyssa Panitch ${ }^{1, *}$ \\ ${ }^{1}$ Weldon School of Biomedical Engineering, Purdue University, West Lafayette, IN 47907
}

\section{Abstract}

Glycosaminoglycans (GAGs) compose one of four classes of mammalian biopolymers, and are arguably the most complex. The research areas of glycobiology, glycopolymers, and the use of GAGs within tissue engineering and regenerative medicine have grown exponentially during the past decade. Researchers are closing in on high throughput methods for GAG synthesis and sequencing, but our understanding of glycan sequence and the information contained in this sequence lags behind. Screening methods to identify key GAG-biopolymer interactions are providing insights into important targets for nanomedicine, regenerative medicine, and pharmaceutics. Importantly, GAGs are most often found in the form of glycolipids and proteoglycans. Several studies have shown that the clustering of GAGs, as is often the case in proteoglycans, increases the affinity between GAGs and other biopolymers. In addition, GAG clustering can create regions of high anionic charge, which leads to high osmotic pressure. Recent advances have led to proteoglycan mimics that exhibit many of the functions of proteoglycans including protection of the extracellular matrix from proteolytic activity, regulation of collagen fibril assembly on the nanoscale, alteration of matrix stiffness, and inhibition of platelet adhesion, among others. Collectively, these advances are stimulating possibilities for targeting of drugs, nanoparticles, and imaging agents, opening new avenues for mimicking nanoscale molecular interactions that allow for directed assembly of bulk materials, and providing avenues for the synthesis of proteoglycan mimics that enhance opportunities in regenerative medicine.

\section{Introduction}

As scientists and engineers seek to unmask the secrets of biopolymers, there is a push to adapt this knowledge to generate materials with predefined complex chemical and physical properties. Since the 1940s, when Erwin Chargraff first noted that adenine and thymine, as well as cytosine and guanine, were found in equivalent ratios in DNA, we have made great headway in understanding DNA. Not only are we now able to precisely sequence and synthesize DNA, but we have also harnessed our understanding of DNA base pairing and are able to make complex materials that assemble with fidelity based upon the encoded nucleic acid sequence. Scientists have accomplished similar feats with RNA. Proteins, which display greater chemical diversity, have also been used to design materials with defined properties, including elastomeric protein polymers and strong silks. The ability to control nucleic acid and amino acid sequence has provided researchers with a rich tool kit with which to design and synthesize multifunctional synthetic materials. More recently, there has been an increased focus on developing methods to synthesize, sequence, and mimic glycosaminoglycans (GAGs) and proteoglycans (PGs) to expand the diversity of our biomimetic tools.

*To whom correspondence should be addressed: Alyssa Panitch, Purdue University, Weldon School of Biomedical Engineering, 206 S. Martin Jischke Drive, West Lafayette, IN 47907-2032, Ph: (765) 496-1313, Fax: (765) 496-1459, apanitch@ purdue.edu. 
Glycosaminoglycans represent the next level of complexity in biopolymers. Mammalian GAGs are composed primarily from glucuronic acid (GlcA), its epimer iduronic acid (IdoA), and $\mathrm{N}$-acetylgalactosamine (GalNAc) sugar monomers. These monomers have variable sulfation patterns, where GlcA is often sulfated on carbon 2 and/or 3 and GalNAc is often sulfated on carbon 4 and/or 6 . While DNA and RNA are codes that can be deciphered to determine their exact sequence and the sequence of the polypeptides that they encode, no such code is apparent for the glycosaminoglycans. The lack of a known glycan code complicates our understanding of the chemical and biophysical significance of the saccharide monomer sequence and sulfation patterns exhibited by GAGs. Thus, this is a major area of research in select laboratories. It is well known that glycan-protein interactions are composed of multiple weak bonds, including electrostatic and hydrogen, and hydrophobic interactions. As such, glycan interactions tend to be multivalent, which ensures strong molecular interactions and provides diversity, ultimately allowing specific ligandreceptor interactions to occur between GAGs and proteins.

Despite the vast amount of research performed to define sugar residues and the variations that exist within these monomers, researchers have not yet identified methods to readily sequence or synthesize GAGs of appreciable length. What is more, while researchers have made great advances in our ability to synthesize short to mid-length oligosaccharides, $, 1,2$ perhaps more challenging is the fact that most GAGs are found pendant to a protein or lipid core, which further modulates function and complicates synthesis. A brief introduction to GAGs, their roles in biological systems, and the field of glycomics was written by Hart and Copeland ${ }^{3}$ the reader is referred to this treatise for additional information on this topic.

Over the course of the last decade, there has been an explosion in the number of manuscripts describing GAG synthesis, utilization of GAGs in materials, and even synthesis of GAG mimics. As such, there exist a large number of recent reviews describing methods used in glycopolymer synthesis, and in some cases, their use in regenerative medicine applications. Table 1 lists reviews of synthetic methods for glycopolymers; for additional information on this subject, please refer to these excellent works. Reviews exist on the modification of natural polysaccharides, including chondroitin and hyaluronan, to make mimics of sulfated GAGs, and of methods for end-functionalization of GAGs to graft them to surfaces and other species. A summary of these reviews follows the glycopolymer reviews in Table 1. Finally, there are several recent reviews of the GAGs themselves and their use as surface coatings and as components of materials. Several of these reviews are listed in Table 2. While blends of GAGs and other biopolymers is also a significant area of research, we have not included this topic in our review and have again provided suggested readings in Table 3 for those interested in this topic.

\section{Glycomimetics}

With respect to investigating chemistry and bioactivity, two major themes emerge from the area of glycomimetic polymers. Researchers are developing techniques to improve our understanding of how monomer sequence and sulfation patterns affect function, as well as improving methodology to synthesize precise motifs. At the same time, others are developing glycopolymers that display multiple monomers or multiple short oligomers in an attempt to mimic and better understand the multivalent interactions that occur between GAGs and their binding partners. Convergence of improved chemistries to mimic GAGs and enhanced understanding of GAG monomer sequence and sulfation patterns should result in glycopolymers with well-defined chemistries and biological activity.

Work in the Hsieh-Wilson, Linhardt, and Seeberger laboratories, among others, seeks to identify how sulfation patterns and glycan clustering contribute to biological activity. Lee et 
al. examined the synthesis of polymer chains with a stiff norborene backbone and pendant chondroitin sulfate-disaccharides, displaying either unsulfated or natural sulfation patterns. ${ }^{4}$ Upon validating that multiple disaccharides pendant to the same polymer backbone bind to antibodies raised against defined sulfation patterns exhibited by chondroitin sulfate, it was then demonstrated that multiple disaccharides bind more strongly to their target proteins than do individual disaccharides. Moreover, the work emphasizes the biological benefits of clustering multiple GAG chains within proteoglycans to strengthen interactions. This concept is consistent with work by Raman and Sasisekharan showing that polyamindoamine dendrimers displaying multiple copies of a heparin/heparin sulfate hexasaccharide inhibits CCL21-mediated leukocyte chemotaxis, while single copies of the hexasaccharide do not. ${ }^{5}$ Utilizing natural GAGs, Tomita, et al. developed a proteoglycan mimic by conjugating both chondroitin sulfate and lipid to a macrocyclic calix[4]resorcarene. ${ }^{6}$ In this construct, they showed that clustering of the chondroitin sulfate chains effectively suppressed cell adhesions, while individual chondroitin sulfate chains per ring were unable to affect adhesion. Collectively, this work shows the importance of multidentate binding in GAG interactions. Drawing inspiration from the aforementioned works, the development of nanomaterials and nanoparticles displaying clusters of GAGs or GAG-binding partners could be developed to take advantage of interactions mediated through multidentate binding. To better control function of these nanomaterials and nanoparticles, key features such as GAG length, monomer identity, and sulfation patterns should be fine-tuned to ensure that the nanomaterials are blood compatible and targeted.

Methods to identify GAG motifs and their associated binding partners will not only improve our understanding of GAG sequence and structure, but also potentially help identify drugable targets. For example, the Lindhart laboratory demonstrated that a defined pentamer sequence from heparin binds with the strongest affinity to antithrombin III and to peptides with similar basic and hydrophobic patterns as the antithrombin III heparin-binding motif. ${ }^{7}$ This finding paved the way for the use of synthetic heparins and low molecular weight heparins as anticoagulants. To help further decode the complexities of glycan structure and sequence, the oligosaccharide array is one method currently being utilized. A review by de Paz and Seeberger discusses the utility of oligosaccharide microarrays for identification of oligosaccharide sequences and sulfation patterns that bind specifically to target molecules to provide insights into material and drug design. ${ }^{8}$ Arrays are not simply limited to short synthetic GAGs. Recently, Takada et al developed an array of various chondroitin sulfates, heparin, and heparin sulfates by end-linking GAGs to a treated slide surface. They validated that the array could distinguish between GAG-growth factor and GAG-antibody interactions, which suggest that the arrays can be used for high-throughput screening to identify GAG binding partners. ${ }^{9}$ Glycopolymer arrays have also been used to identify interactions between individual and clusters of GAG monomers and dimers. ${ }^{4}$ These high throughput methods increase our depth of knowledge about GAGs and increase our molecular tool kit for screening and for design of biomimetic materials.

While there is clear evidence that sulfation patterns within GAG chains are critical for certain biological activities, it is also evident that sulfation density plays an important role in binding to molecules known to interact with GAGs. Although the glycopolymer and sulfated polymer interactions may not be as specific or as strong as those seen between natural GAGs and proteins, they may be important in the development of biomaterials for various applications including controlled release, and antithrombotic and anti-inflammatory therapies. Recent work in the Haag laboratory using several glycan monomers (galactose, mannose, fructose, $\mathrm{N}$-acetylglucosamine, and lactose) conjugated pendant to hyperbranched polyglycerol indicated that while clustering of the glycan monomers can, in some cases, improve binding to selectins, addition of sulfates to the glycans appeared to be more important to enhanced binding. ${ }^{10}$ Selectins are cell receptors that bind to saccharide motifs 
during an early step in leukocyte adhesion. As such, there is significant work being performed to mimic selectin binding to newly develop treatments in order to control selectin-leukocyte interactions and inflammation. In light of the importance of multidentate binding in many GAG interactions, it is surprising that clustering was not found to be significantly important in the above work, however the authors note that alternate conjugation chemistries may be needed to allow more flexibility as the saccharide units bind to the selectin receptors.

The importance of sulfation becomes even more apparent when investigating the fabrication of synthetic polymers with glycan-like features. Oezyuerek, et al. modified pNIPAm with pendant glycan groups, which were further tailored to include sulfate moieties, to generate heparin-like polymers. ${ }^{11}$ The polymers were surface-grafted to control growth factor adsorption and cell adhesion to surfaces. As expected, the addition of sulfate moieties to the glycomimetic polymers increased the adsorption of growth factor and also aided in the adhesion and proliferation of human umbilical vein endothelial cells. Due to the antithrombotic nature of many sulfated GAGs, this work suggests that glycol-polymer mimetics will compose useful materials as we develop new blood contacting surfaces and tissue engineered constructs.

Cerrada, et al. utilized an ethylene-vinyl alcohol copolymer to generate lactone-based glycopolymers. The glycopolymers contained the pendant aminosaccharide groups $\mathrm{N}$-(4aminobutyl)-D-gluconamide (NABG), N-(4aminobutyl)-O- $\beta$-D-galactopyranosyl-(1-4)-Dgluconamide (NABL), and $\mathrm{N}$-(4aminobutyl)-O- $\beta$-D-glucopyranosyl-(1-4)-D-gluconamide (NABM). The researchers found that NABM compounds bound to concanavalin A, a lectin known to bind to mannosyl and glucosyl residues, while NABL containing polymers bound to $\mathrm{RCA}_{120}$, a lectin with high affinity for $\beta$-D-galactosyl residues. Thus, utilizing an ethyleneoxide backbone with glycan modification, Cerrada, et al. developed a system to specifically bind to, or potentially detect, various glycan-binding biopolymers. ${ }^{12}$

In an attempt to mimic branching observed in some polysaccharides and to provide multiple GAG presentation points per hydrophilic polymer chain, Ahmed, et al. generated hyperbranched 3-gluconamidopropylmethacrylate and 2-

lactobionaminoethylmethacrylamide polymers and investigated their blood compatibility. ${ }^{13}$ Thorough investigation demonstrated that these molecules do not interfere with clotting or complement activity, nor do they promote hemolysis. Thus, these types of systems may be useful for nanomedicine and intravascular drug delivery. Besenius, et al. utilized galactose monomethacrylate and either ethylene glycol dimethacrylate or divinylbenzene to promote branching to create branch glycopolymers. Critical to the synthesis of branched polymers using free radical polymerization was the addition of a chain transfer agent to prevent gelation. ${ }^{14}$ As described in several reviews, significant progress has been made in the synthesis of branched and dendritic polymers to develop methods to mimic the valency seen in many GAGs and proteoglycans, as well as in other important polysaccharides found in nature (Table 1). These glycopolymers aim to mimic many of the important functions of mammalian GAGs and proteoglycans including growth factor binding and release, cell binding and recognition, and a vast number of ligand interactions. For additional information in current development of glycopolymers for use as biomaterials, the reader is referred to a recent review by Minura. ${ }^{15}$

\section{Modifications to Natural GAGs}

While the precise fabrication of synthetic glycopolymers allows for bulk fabrication of bioactive polymers with well-defined activities, creating these molecules with both high reproducibility and yield has yet to be achieved. In addition, unlike with DNA, RNA, and 
proteins, we do not yet have a sufficient understanding of how nature controls the sequencing. Thus, the manipulation of natural GAGs to meet the growing demand for bioactive GAG materials is of interest to many researchers. It has previously been shown that simple modifications of GAGs permit researchers to tailor their activity; for instance, slight chemical alterations to hyaluronan allow for the extended residence time of the polysaccharide within the body. ${ }^{16}$

The modification of natural GAG structures can also be used to obtain variations of GAGs that are difficult to isolate from tissue sources. Previously, chondroitin sulfate-E (chondroitin-4,6-disulfate) has been successfully prepared from chondroitin sulfate-A (chondroitin-4-sulfate) via regioselective sulfonation. ${ }^{17}$ The technique utilized not only provided an economical means of preparing rare chondroitin sulfate-E from a more readily available chondroitin sulfate-A, but also showed that interactions of the synthetic chondroitin sulfate-E with fibroblast growth factor (FGF) were very similar to the binding kinetics and binding affinities exhibited by natural chondroitin sulfate-E. To demonstrate that chondroitin sulfate variants can be generated in high yield for microbial organisms, Bendini, et al describe the modification of chondroitin, a GAG isolated from the bacterium E. coli O5:K4:H4. ${ }^{18}$ Mild hydrolysis removed $\beta$-fructose residues from the GlcA O-3 position to yield chondroitin, which was further modified to yield various unnatural and rare forms of chondroitin sulfate. The chemical means of protection and sulfation are leading us closer to methods to mass-produce natural forms of chondroitin sulfate. While these methods provide a renewable source of chondroitin sulfate, they do not provide the control over chain length and chemical fingerprint that will aid in defining how these features contribute to biological function, including receptor ligand binding. Knowledge of these features is critical if we are to unleash the full potential of these molecules for smart materials design such as that achieved with nucleic acids and amino acids.

The methods required to modify the chemical structure of GAGs in a precise manner to gain better control over desired glycan-protein interactions remains a main focus with respect to GAG research. Ultimately, combined knowledge of GAG-protein interactions, comprehension to fine tune these interactions with regard to binding affinity, and methods to synthesize and modify defined GAG sequence will allow us to develop targeting strategies for nano- and micro-particles, well-defined controlled release vehicles, and molecular therapeutics.

Heparin is one of the most studied GAGs and a plethora of information shows that a major driving force for interactions with proteins stems from its anionic nature. This GAG naturally exhibits a high negative charge density that results from approximately 2.7 sulfate groups per disaccharide unit ${ }^{19}$, and enables interactions with several proteins, such as growth factors, cytokines, and proteases. Heparin interacts with various proteins with different affinities and is a prime example of how biology utilizes GAG sequencing and sulfation to control molecular interactions. In order to mimic the molecular interactions exhibited by heparin, efforts, similar to those described above for the modification of chondroitin and hyaluronan, have been made to investigate the chemical modification of other GAGs.

Upon examining the over-sulfation of dermatan sulfate, chondroitin sulfate, and heparin sulfates, it was reported that over-sulfated GAGs exhibited enhanced FGF affinity and FGF receptor signaling, compared to unmodified GAGs ${ }^{20}$ Similarly, Satoh, et al demonstrated that the addition of sulfur groups to hyaluronan allowed for increased affinity to proteins, such as fibronectin, a feat that remains unattainable with unmodified hyaluronan. ${ }^{21}$ Furthermore, by controlling the degree of hyaluronan sulfation, mixtures of various proteins were purified due to the variable potency of their binding interactions with the over-sulfated 
hyaluronan. Thus, the modulation of sulfur moieties on GAGs allows for tuning proteinGAG interactions, to give rise to low, moderate, or high affinity interactions.

Freeman, et al recently demonstrated the powerful binding effects of sulfur-modified hyaluronan molecules, showing that the binding affinity of FGF to the sulfur-modified hyaluronan was a full magnitude greater than the affinity interactions that occur between FGF and heparin. ${ }^{22}$ Other researchers have reported this same phenomenon, which could have many important implications in drug delivery applications. ${ }^{23}$ The use of modified GAGs within nanoparticle systems allows for improved drug loading and delivery. While there is a strong focus on increasing the sulfate content of GAGs in order to strengthen known GAG-protein interactions, GAGs can also be modified to be cationic in order to tailor their interactions with anionic therapeutics. Recently, Ilgin, et al synthesized nanoparticles from hyaluronan modified to have a positive charge. ${ }^{24}$ Due to the positive nature of the modified hyaluronan, an increased amount of drug compound, which exhibited a slight negative charge, was loaded in the nanoparticles and drug release was slower compared to non-modified hyaluronan particles. This same interaction could be utilized to control the release of positively charged drug compounds from nanoparticles composed of over-sulfated GAGs molecules.

The modification of GAGs not only impacts glycan-protein interactions, but can also have substantial impacts on cell function. Moller, et al recently examined the effects of hyaluronan derivatives against HSV-1 in cells, demonstrating that increased sulfation on hyaluronan led to lower concentrations of hyaluronan required to inhibit the HSV-1 induced cytopathic effect. ${ }^{25}$ While carboxymethylated hyaluronan derivatives did not enhance antiviral activity, the addition of carboxymethylate moieties to sulfate-modified hyaluronan did not inhibit antiviral activity. Alternatively, it is plausible to over-modify hyaluronan such that its biological activity is decreased. To add biological stimuli to tissue engineered constructs, Eng, et al crosslinked thiolyated hyaluronan with poly(ethylene glycol) (PEG) diacrylate. ${ }^{26}$ As the degree of hyaluronan thiolation increased, decreases in hyaluronan biological activity where exhibited, where both reduced susceptibility to hyaluronidase and neurite outgrowth were observed.

The modification of GAGs to control cell function has been a major focus within the field of tissue-engineered constructs. Covalent incorporation of GAGs within biological scaffolds has been shown to provide mechanical support, ${ }^{27}$ act as chemical signals for cells, ${ }^{28}$ and aid in the controlled release of growth factors and drug compounds. ${ }^{29}$ Common modifications of GAGs, including the addition of methacrylate, ${ }^{30}$ aldehyde, ${ }^{31}$ or thiol ${ }^{32}$ moieties, allow for covalent crosslinking of GAGs into constructs.

Alternatively, non-covalent assembly of hydrogels is also possible through affinity crosslinking that naturally occurs between GAGs and proteins. Utilizing heparin-binding peptides conjugated to PEG star polymers, Seal and Panitch showed that peptide-GAG interactions could be used to assemble gel-like materials. Taking advantage of both the assembly properties of GAGs and proteins, and capitalizing on the ability to utilize GAGgrowth factor interactions to control the release of vascular endothelial growth factor, the Kiick group demonstrated the successful assembly of PEG-based hydrogels. ${ }^{29}, 33$ In the latter work, star-PEG was conjugated to heparin or to heparin-binding peptides and the two components were mixed to induce assembly; where as in the former study, only peptide was conjugated to PEG, while the heparin was unmodified. Similarly, Tan, et al created hydrogels via affinity crosslinking between low molecular weight heparin that was tethered to hyaluronan and a peptide derived from heparin interacting protein (HIP), which was bound to 4-arm PEG. ${ }^{34}$ Hydrogels containing linked heparin-hyaluronan molecules exhibited increased stiffness and as the gel disassembled, HIP was released over a period of 
14 days. Thus, the inclusion of GAGs within these types of tissue-engineered scaffolds not only aids in their assembly, but also serves as a therapeutic delivery mechanism. Looking forward, the use of modified GAGs within these systems may lead to more controlled release profiles and the ability to release multiple cell signaling molecules over time.

It should be noted that while manipulating the structure of GAGs can allow for the better control of cell function within synthetic systems, it can also lead to unexpected, and sometimes undesirable, consequences. While increasing the sulfur content of HA has been shown to encourage growth factor binding, Satoh, et al reported that sulfur-rich hyaluronan led to a decrease in keratinocyte proliferation and adhesion, compared to unmodified hyaluronan. ${ }^{35}$ Furthermore, in an effort to transform heparin sulfate such that it better resembled the growth factor-binding properties of heparin, Papy-Garcia, et al increased the sulfur content of heparin sulfate. ${ }^{36}$ While the modified heparin sulfate was able to bind to VEGF more potently than heparin, it also resulted in decreased cell proliferation. This suggests that too great an increase in the binding affinity between polymer and growth factor may reduce availability and biological activity of the growth factor. As such, it is imperative to balance enhanced biological activity with over-modification in order to synthesize useful GAGs.

\section{Mimicry of Proteoglycans and Glycolipids}

While GAG chemistry itself is sufficiently complex, mammalian GAGs are often found conjugated to a protein or lipids, thus broadening the complexity and function of these molecules. Synthetic methods that lead to fabrication of molecules that mimic the function of glycolipids and proteoglycans will enhance our ability to generate biomimetic nanostructures and materials.

In order to fabricate these biomimetic molecules, natural GAGs can be modified with a variety of functional moieties in order to aid in the attachment of lipids or proteins. For example, Ruhela, et al noted that high molecular weight HA could be lysed into a low molecular weight form with a functional aldehyde end group, thus enabling coupling of the HA derivative to lipids or proteins to create more complex macromolecules ${ }^{37}$ Expanding upon this concept, simple forms of proteoglycans have been produced by merely tethering BSA to either heparin or chondroitin sulfate via 1-ethyl-3-(3dimethylaminopropyl)carbodiimide (EDAC) modification. ${ }^{38}$ Interestingly, an in vitro application of these simple proteoglycan-mimics resulted in decreased viability of several cancer cell lines, compared to treatment with GAGs only. This phenomenon occurred even in the presence of GAG-degrading enzymes. It should be noted that these mimics are not cell specific and as such, are also capable of causing apoptosis in non-tumorigenic cells lines. However, the work demonstrates facile methods to conjugate GAGs to proteins and the dramatic difference seen in biological activity when GAGs are presented as ungrafted polymers as compared to grafted forms that mimic proteoglycans.

The creation of a polysaccharide-protein mimic to simulate the natural proteoglycan aggrecan has been of high interest for many researchers. Interestingly enough, Han, et al recently reported that adding HA and link protein to aggrecan allows for the biomimetic reassembly of proteoglycan aggregates ${ }^{39}$. Furthermore, the reassembly of these macromolecules enhanced the compressive properties of hydrogels, in addition to promoting viable chondrocytes capable of expressing collagen II and GAGs. Thus, it has been of great interest to many research groups to develop an aggrecan mimic, which demonstrates similar characteristics.

Jin, et al reported the synthesis of a polysaccharide hybrid displaying a macromolecular structure similar to that of the proteoglycan aggrecan. ${ }^{40}$ This mimic, consisting of tyramine- 
conjugated dextran chains grafted to hyaluronan backbones, was utilized to create hydrogel networks able to sustain viable chondrocytes and promote GAG and collagen production. While this molecule mimicked the structure of aggrecan by linking multiple dextran molecules to one hyaluronan molecule, the Marcolongo group has taken another approach to mimicking aggrecan. Due to the density of end-linked chondroitin sulfate in aggrecan, the Marcolongo group developed a method to functionalize chondroitin-4-sulfate at the reducing end and incorporate it into poly(acrylic acid). In this way, they have generated bottle-brush polymers that are rich in chondroitin sulfate. Through extensive characterization they have shown that on a $250 \mathrm{kDa}$ poly(acrylic acid) backbone they could graft $\sim 85 \mathrm{CS}$ chains, creating a molecule with an overall molecular weight of $\sim 2,000 \mathrm{kDa}$. These constructs achieved an osmotic pressure equivalent to that seen with natural aggrecan. Interestingly, they also found that properties scale to molecular size, thus be creating constructs with short PAA backbones and fewer CS chains, they lowered the overall molecular weight and osmotic pressure achieved by the aggrecan mimics. ${ }^{41}$

Alternatively, we, and others, have taken the approach of focusing on fabricating molecules that mimic the function of proteoglycans, but may not exhibit similar structural characteristics. For example, we have demonstrated that by conjugating one or more collagen-binding peptides to the backbone of dermatan sulfate, we can generate a mimic of the small leucine-rich proteoglycan decorin. ${ }^{42}$ Similar to decorin, these molecules influence the fibril diameter of polymerizing type I collagen on the nano-scale. Moreover, they influence the stiffness of collagen-rich tissue mimics and protect collagen from proteolytic degradation, a phenomenon that was not achieved with dermatan sulfate alone. These decorin mimics have been used as a molecular, or nanotherapy, to reduce scarring in a rat linear incision model, presumably due both to their ability to influence collagen fibril diameter and to protect collagen from proteolytic degradation. ${ }^{43}$

Using a similar strategy, Bernhard and Panitch have developed a functional mimic of aggrecan. ${ }^{44}$ Rather than develop a large bottlebrush construct, they decorated the backbone of chondroitin-6-sulfate with hyaluronan-binding peptides. These functional aggrecan mimics increased the compressive strength of collagen gels, as well as protected the gels from proteolytic degradation. Sharma, et al further showed that unlike unmodified hyaluronan and chondroitin sulfate, the aggrecan mimics promoted both type II collagen and aggrecan expression and suppressed proteolytic enzyme expression when encapsulated with primary bovine chondrocytes within collagen gels. ${ }^{45}$ In unpublished work, Sharma and Panitch demonstrated that the mimics were able to penetrate into cartilage, and like aggrecan itself, the mimics protected the cartilage from proteolytic degradation and contributed to the overall compressive stiffness of the cartilage.

The Bertozzi laboratory described pioneering work where they conjugated mono- or disaccharides to a hydrophobic tail to generate a mucin mimic with $\mathrm{N}$-acetylgalactosamine sugars. ${ }^{46}$ In this work, they demonstrated good incorporation of the mucin mimics into the cell membrane of a mucin deficient $I d D$ CHO cell line. They further verified specific glycolipid-protein interactions occurring between cell-embedded mucins containing $\alpha-\mathrm{N}$ acetylgalactosamine and Heilix Pomatia agglutinin, but not with the mucin made with $\beta$ - Nacetylgalactosamine. Their work opens up the opportunity to study cell-substrate and cellcell interactions and the impact of specific GAGs on those interactions.

In an attempt to mimic the antithrombotic function of the glycocalix, Paderi, et al conjugated multiple collagen-binding peptides to a dermatan sulfate backbone, with goals of taking advantage of the anti-platelet function of dermatan sulfate and the ability of collagenbinding peptides to bind to collagen. ${ }^{47}$ The therapeutic molecules were delivered to blood vessels following balloon angioplasty to demonstrate that the conjugate could bind to the 
exposed collagen found on the interior of the injured vessel. It was reported that like the glycocalyx, this mimic prevented platelet binding and activation on the collagen, thus suppressing any resultant intimal hyperplasia that would normally occur sans mimic.

\section{Conclusions \& Future Outlooks}

It is evident from the research described in this review that by mimicking GAG, glycolipid and proteoglycan function, researchers have developed tools to better understand biological function and developed potential molecular therapeutics to improve human health. Proteoglycans take advantage of nanoscale interactions used in biology to build complex structures, convey and dissipate biochemical signals, and transmit mechanical stresses. While it remains difficult to precisely synthesize GAGs, as is done with DNA, RNA and protein, synthetic glycopolymers and natural GAGs remain viable alternatives with respect to development of glycopolymer and proteoglycan mimics that convey much of the biological activity of their native counterparts. These mimics can replace native GAG structure and are being investigated as therapeutics to improve tissue function and healing. In the mean time, rapid progress in synthetic GAG methods and GAG sequencing will increase our understanding of the nanoscale interactions that give rise to the vast number of protein-GAG, GAG-GAG, and GAG-lipid interaction that drive many biological activities. This improved understanding will drive next generation glycol-therapies, molecular and nanoparticle targeting modalities, biomimetic materials, both bulk and nanoparticle, and provide a means to identify new drugable targets.

Importantly, as researchers design new nanomaterials and nanoparticles we can immediately take advantage of many of the lessons already learned from the exploration of GAG structure and activity. Nanoparticles can display clusters of GAGs or GAG-binding partners to take advantage of the multidentate binding to sequester nanoparticles on or within tissues; sequestering the particles within tissues can improve local drug delivery, or enhance image contrast for particles designed for imaging. Key features such as GAG length, monomer identity and sulfation can also be used to enhance targeting, influence tissue binding, induce hemocompatibility, and control the release of therapeutic agents. It may also be possible to learn from nature to control fibril diameter and self-assembly. While a decorin mimic was developed to influence collagen fibrillogenesis and to protect collagen fibrils from degradation, these concepts can be extended to other biopolymers or even to synthetic polymer nanofibers. As with DNA, RNA, and proteins, there is much to learn from GAG structure and function to improve materials for nanomedicine.

\section{Acknowledgments}

R.S. is supported by a NSF Graduate Research Fellowship. Funding for this research was through NIH R01HL106792.

\section{References}

1. Larsen K, Thygesen MB, Guillaumie F, Willats WGT, Jensen KJ. Solid-phase chemical tools for glycobiology. Carbohydrate Research. 2006; 341:1209-1234. [PubMed: 16716275]

2. Noti C, Seeberger PH. Chemical Approaches to Define the Structure-Activity Relationship of Heparin-like Glycosaminoglycans. Chemistry and Biology. 2005; 12:731-756. [PubMed: 16039522]

3. Hart GW, Copeland RJ. Glycomics Hits the Big Time. Cell. 2010; 143:672-676. [PubMed: 21111227]

4. Lee SG. End-functionalized glycopolymers as mimetics of chondroitin sulfate proteoglycans. Chemical Science. 2010; 1:322. [PubMed: 21274421] 
5. Raman R, Sasisekharan R. Cooperativity in glycan-protein interactions. Chemical Biology. 2007; 14:873-874.

6. Tomita N, Sando S, Sera T, Aoyama Y. Macrocyclic proteoglycan mimics,Potent inhibition of cell adhesion by a bundle of chondroitin sulfate chains assembled on the calix[4]resorcarene platform. Bioorganic and Medicinal Chemistry Letters. 2004; 14:2087-2090. [PubMed: 15080984]

7. Bae J, Desai UR, Pervin A, Caldwell EE, Weiler JM, Linhardt RJ. Interaction of heparin with synthetic antithrombin III peptide analogues. Biochemical Journal. 1994; 301:121-129. [PubMed: 8037658]

8. de Paz JL, Seeberger PH. Deciphering the glycosaminoglycan code with the help of microarrays. Molecular BioSystems. 2008; 4:707-711. [PubMed: 18563243]

9. Takada W, Fukushima M, Pothacharoen P, Kongtawelert P, Sugahara K. A sulfated glycosaminoglycan array for molecular interactions between glycosaminoglycans and growth factors or anti-glycosaminoglycan antibodies. Analytical Biochemistry. 2013; 435:123-130. [PubMed: 23333222]

10. Dernedde J, Papp I, Enders S, Wedepohl S, Paulus F, Haag R. Synthesis and Evaluation of Nonsulfated and Sulfated Glycopolymers as L- and P-selectin Inhibitors. Journal of Carbohydrate Chemistry. 2011; 30:347-360.

11. Oezyuerek Z, Franke K, Nitschke M, Schulze R, Simon F, Eichhorn KJ, Pompe T, Werner C, Voit B. Sulfated glyco-block copolymers with specific receptor and growth factor binding to support cell adhesion and proliferation. Biomaterials. 2009; 30:1026-1035. [PubMed: 19058844]

12. Cerrada ML, Ruiz C, Sánchez-Chaves M, Fernández-García M. Molecular recognition capability and rheological behavior in solution of novel lactone-based glycopolymers. European Polymer Journal. 2009; 45:3176-3186.

13. Ahmed M, Lai BFL, Kizhakkedathu JN, Narain R. Hyperbranched Glycopolymers for Blood Biocompatibility. Bioconjugate Chemistry. 2012; 23:1050-1058.

14. Besenius P, Slavin S, Vilela F, Sherrington DC. Synthesis and characterization of water-soluble densely branched glycopolymers. Reactive and Functional Polymers. 2008; 68:1524-1533.

15. Miura Y. Synthesis and biological application of glycopolymers. Journal of Polymer Science Part A: Polymer Chemistry. 2007; 45:5031-5036.

16. Hahn SK, Jelacic S, Maier RV, Stayton PS, Hoffman AS. Anti-inflammatory drug delivery from hyaluronic acid hydrogels. Journal of Biomaterials Science, Polymer Edition. 2004; 15:11111119. [PubMed: 15503629]

17. Cai C, Solakyildirim K, Yang B, Beaudet JM, Weyers A, Linhardt RJ, Zhang F. Semi-synthesis of chondroitin sulfate-E from chondroitin sulfate-A. Carbohydrate Polymers. 2012; 87:822-829. [PubMed: 22140285]

18. Bedini E, De Castro C, De Rosa M, Di Nola A, Restaino OF, Schiraldi C, Parrilli M. SemiSynthesis of Unusual Chondroitin Sulfate Polysaccharides Containing GlcA(3-O-sulfate) or GlcA(2,3-di-O-sulfate) Units. Chemistry - A European Journal. 2012; 18:2123-2130.

19. Capila I, Linhardt RJ. Heparin-Protein Interactions. Angewandte Chemie International Edition. 2002; 41:390-412.

20. Pan J, Qian Y, Zhou X, Lu H, Ramacciotti E, Zhang L. Chemically Oversulfated Glycosaminoglycans Are Potent Modulators of Contact System Activation and Different Cell Signaling Pathways. Journal of Biological Chemistry. 2010; 285:22966-22975. [PubMed: 20418371]

21. Satoh T, Nishiyama K, Nagahata M, Teramoto A, Abe K. The research on physiological property of functionalized hyaluronan: interaction between sulfated hyaluronan and plasma proteins. Polymers for Advanced Technologies. 2004; 15:720-725.

22. Freeman I, Kedem A, Cohen S. The effect of sulfation of alginate hydrogels on the specific binding and controlled release of heparin-binding proteins. Biomaterials. 2008; 29:3260-3268. [PubMed: 18462788]

23. Cai S, Liu Y, Zheng Shu X, Prestwich GD. Injectable glycosaminoglycan hydrogels for controlled release of human basic fibroblast growth factor. Biomaterials. 2005; 26:6054-6067. [PubMed: 15958243] 
24. Ilgin P, Avci G, Silan C, Ekici S, Aktas N, Ayyala RS, John VT, Sahiner N. Colloidal drug carries from (sub)micron hyaluronic acid hydrogel particles with tunable properties for biomedical applications. Carbohydrate Polymers. 2010; 82:997-1003.

25. Möller S, Schmidtke M, Weiss D, Schiller J, Pawlik K, Wutzler P, Schnabelrauch M. Synthesis and antiherpetic activity of carboxymethylated and sulfated hyaluronan derivatives. Carbohydrate Polymers. 2012; 90:608-615.

26. Eng D, Caplan M, Preul M, Panitch A. Hyaluronan scaffolds: A balance between backbone functionalization and bioactivity. Acta Biomaterialia. 2010; 6:2407-2414. [PubMed: 20051273]

27. Li Q, Williams CG, Sun DDN, Wang J, Leong K, Elisseeff JH. Photocrosslinkable polysaccharides based on chondroitin sulfate. Journal of Biomedical Materials Research Part A. 2004; 68A:28-33. [PubMed: 14661246]

28. Nie T, Akins RE, Kiick KL. Production of heparin-containing hydrogels for modulating cell responses. Acta Biomaterialia. 2009; 5:865-875. [PubMed: 19167277]

29. Zhang L, Furst EM, Kiick KL. Manipulation of hydrogel assembly and growth factor delivery via the use of peptide-polysaccharide interactions. Journal of Controlled Release. 2006; 114:130-142. [PubMed: 16890321]

30. Benoit DSW, Anseth KS. Heparin functionalized PEG gels that modulate protein adsorption for hMSC adhesion and differentiation. Acta Biomaterialia. 2005; 1:461-470. [PubMed: 16701827]

31. Aulin C, Bergman K, Jensen-Waern M, Hedenqvist P, Hilborn J, Engstrand T. In situ crosslinkable hyaluronan hydrogel enhances chondrogenesis. Journal of Tissue Engineering and Regenerative Medicine. 2011; 5:188-196.

32. Tae G, Kim Y-J, Choi W-I, Kim M, Stayton PS, Hoffman AS. Formation of a Novel HeparinBased Hydrogel in the Presence of Heparin-Binding Biomolecules. Biomacromolecules. 2007; 8:1979-1986. [PubMed: 17511500]

33. Yamaguchi N, Zhang L, Chae B-S, Palla CS, Furst EM, Kiick KL. Growth Factor Mediated Assembly of Cell Receptor-Responsive Hydrogels. Journal of the American Chemical Society. 2007; 129:3040-3041. [PubMed: 17315874]

34. Tan H, Zhou Q, Qi H, Zhu D, Ma X, Xiong D. Heparin Interacting Protein Mediated Assembly of Nano-Fibrous Hydrogel Scaffolds for Guided Stem Cell Differentiation. Macromolecular Bioscience. 2012; 12:621-627. [PubMed: 22454284]

35. Satoh T, Nagahata M, Teramoto A, Hatimori A, Abe K, Im SS. The basic research on physiological property of functionalized hyaluronan-I. Effect of hyaluronan and sulfated hyaluronan on cell proliferation of human epidermal keratinocytes. Polymers for Advanced Technologies. 2004; 15:329-334.

36. Papy-Garcia D, Barbier-Chassefière V, Rouet V, Kerros M-E, Klochendler C, Tournaire MC, Barritault D, Caruelle JP, Petit E. Nondegradative Sulfation of Polysaccharides. Synthesis and Structure Characterization of Biologically Active Heparan Sulfate Mimetics. Macromolecules. 2005; 38:4647-4654.

37. Ruhela D, Riviere K, Szoka FC. Efficient Synthesis of an Aldehyde Functionalized Hyaluronic Acid and Its Application in the Preparation of Hyaluronan-Lipid Conjugates. Bioconjugate Chemistry. 2006; 17:1360-1363. [PubMed: 16984148]

38. Pumphrey CY, Theus AM, Li S, Parrish RS, Sanderson RD. Neoglycans, Carbodiimide-modified Glycosaminoglycans: A New Class of Anticancer Agents That Inhibit Cancer Cell Proliferation and Induce Apoptosis. Cancer Research. 2002; 62:3722-3728. [PubMed: 12097281]

39. Han E, Wilensky LM, Schumacher BL, Chen AC, Masuda K, Sah RL. Tissue Engineering by Molecular Disassembly and Reassembly: Biomimetic Retention of Mechanically Functional Aggrecan in Hydrogel. Tissue Engineering Part C: Methods. 2012; 16:1471-1479. [PubMed: 20486781]

40. Jin R, Moreira Teixeira LS, Dijkstra PJ, van Blitterswijk CA, Karperien M, Feijen J. Enzymatically-crosslinked injectable hydrogels based on biomimetic dextran-hyaluronic acid conjugates for cartilage tissue engineering. Biomaterials. 2010; 31:3103-3113. [PubMed: 20116847] 
41. Prudnikova K, Vresilovic EJ, Marcolongo M. New Strategy for Treatment of Lower Back Pain: Molecular Restoration of Intervertebral Disc Using an Injection of Biomimetic Aggrecan. The Spine Journal. 2012; 12:S30.

42. Paderi JE, Sistiabudi R, Ivanisevic A, Panitch A. Collagen-Binding Peptidoglycans: A Biomimetic Approach to Modulate Collagen Fibrillogenesis for Tissue Engineering Applications. Tissue Engineering Part A. 2009; 15:2991-2999. [PubMed: 19323607]

43. Stuart KA, Paderi JE, Snyder P, Freeman L, Panitch A. Collagen-Binding Peptidoglycans Inhibit MMP Mediated Collagen Degradation and Reduce Dermal Scarring. PLoS ONE. 2011; 6:e22139. [PubMed: 21779387]

44. Bernhard JC, Panitch A. Synthesis and characterization of an aggrecan mimic. Acta Biomaterialia. 2012; 8:1543-1550. [PubMed: 22248525]

45. Sharma S, Panitch A, Neu CP. Incorporation of an aggrecan mimic prevents proteolytic degradation of anisotropic cartilage analogs. Acta Biomaterialia. 2013; 9:4618-4625. [PubMed: 22939923]

46. Saxon E, Bertozzi CR. Chemical and Biological Strategies for Engineering Cell Surface Glycosylation. Annual Review of Cell and Developmental Biology. 2001; 17:1-23.

47. Paderi JE, Stuart K, Sturek M, Park K, Panitch A. The inhibition of platelet adhesion and activation on collagen during balloon angioplasty by collagen-binding peptidoglycans. Biomaterials. 2011; 32:2516-2523. [PubMed: 21216002]

48. Vazquez-Dorbatt V, Lee J, Lin EW, Maynard HD. Synthesis of glycopolymers by controlled radical polymerization techniques and their applications. ChemBioChem. 13:2478-2487. [PubMed: 23132748]

49. Voit B, Appelhans D. Glycopolymers of Various Architectures-More than Mimicking Nature. Macromolecular Chemistry and Physics. 2010; 211:727-735.

50. Vibert A, Jacquinet Js, Lopin-Bon C. Recent Advances in the Chemical and Enzymatic Chondroitin Sulfate Synthesis. Journal of Carbohydrate Chemistry. 2011; 30:393-414.

51. Slavin S, Burns J, Haddleton DM, Becer CR. Synthesis of glycopolymers via click reactions. European Polymer Journal. 2011; 47:435-446.

52. Gemma E, Meyer O, Uhrin D, Hulme AN. Enabling methodology for the end functionalisation of glycosaminoglycan oligosaccharides. Molecular BioSystems. 2008; 4:481-495. [PubMed: 18493641]

53. DeAngelis P. Glycosaminoglycan polysaccharide biosynthesis and production: today and tomorrow. Applied Microbiology and Biotechnology. 2012; 94:295-305. [PubMed: 22391966]

54. Jia X, Kiick KL. Hybrid multicomponent hydrogels for tissue engineering. Macromolecular Bioscience. 2009; 9:140-156. [PubMed: 19107720]

55. Baldwin AD, Kiick KL. Polysaccharide-modified synthetic polymeric biomaterials. Peptide Science. 2010; 94:128-140. [PubMed: 20091875]

56. Hudalla GA, Murphy WL. Biomaterials that regulate growth factor activity via bioinspired interactions. Advanced Functional Materials. 21:1754-1768. [PubMed: 21921999]

57. Ferdous Z, Grande-Allen KJ. Utility and Control of Proteoglycans in Tissue Engineering. Tissue Engineering. 2007; 13:1893-1904. [PubMed: 17518731]

58. Yannas IV, Tzeranis DS, Harley BA, So PT. Biologically active collagen-based scaffolds: advances in processing and characterization. Philosophical Transactions. Series A, Mathematical, Physical, and Engineering Sciences. 2010; 368:2123-2139. 
Table 1

Selected Reviews of Synthetic Methods for Glycopolymers and Modification of Natural Polysaccharides

\begin{tabular}{|c|c|}
\hline Reference & Content \\
\hline \multicolumn{2}{|l|}{ Synthetic Methods for Glycopolymers } \\
\hline $\begin{array}{l}\text { Vazquez-Dorbatt, et al., ChemBioChem, 2012, } \\
13: 2478-2487 .{ }^{48}\end{array}$ & $\begin{array}{l}\text { This review focuses on controlled free radical } \\
\text { polymerization with a focus on polymers with } \\
\text { sugar residues pendant to the backbone. The } \\
\text { topic is covered from a synthesis perspective. }\end{array}$ \\
\hline $\begin{array}{l}\text { Voit and Appelhans, Macromolecular } \\
\text { Chemistry and Physics, 2010, 11:727-735.49 }\end{array}$ & $\begin{array}{l}\text { This review focuses on synthetic methods for } \\
\text { linear and branched glycopolymer synthesis. }\end{array}$ \\
\hline $\begin{array}{l}\text { Bendini and Parrilli, Carbohydrate Research, } \\
\text { 2012, 356:75-85. }\end{array}$ & $\begin{array}{l}\text { This review discusses synthetic methods for } \\
\text { making short chondroitin sulfate oligos and } \\
\text { modification of long chondroitin chains to form } \\
\text { chondroitin sulfate. There is also a brief } \\
\text { review of methods for glycopolymer mimics. }\end{array}$ \\
\hline $\begin{array}{l}\text { Vibbert, Jacquint and Lopin-Bon, Journal of } \\
\text { Carbohydrate Chemistry, 2011, 30:393-414. } .^{50}\end{array}$ & $\begin{array}{l}\text { The authors provide a concise review of } \\
\text { methods for synthesizing short chondroitin } \\
\text { sulfate oligosaccharides and describe } \\
\text { protecting methods to achieve oligos with both } \\
\text { common and rare sulfation patterns. }\end{array}$ \\
\hline $\begin{array}{l}\text { Slavin, et al., European Polymer Journal, 2011, } \\
47: 435-446 .{ }^{51}\end{array}$ & $\begin{array}{l}\text { This review introduces various glucomonomers } \\
\text { and describes the use of click chemistry to } \\
\text { synthesize glycopolymers, with an emphasis on } \\
\text { modifying existing polymers with pendant } \\
\text { sugar residues. }\end{array}$ \\
\hline \multicolumn{2}{|l|}{ Modification of Natural Polysaccharides } \\
\hline $\begin{array}{l}\text { Gemma, et al., Molecular BioSystems, } 2008 \text {, } \\
4: 481-495.52\end{array}$ & $\begin{array}{l}\text { This review describes work done to cleave } \\
\text { GAGs and various methods of GAG end } \\
\text { functionalization. }\end{array}$ \\
\hline $\begin{array}{l}\text { DeAngelis, Applied Microbiology } \\
\text { Biotechnology,2012, 94:295-305. }{ }^{53}\end{array}$ & $\begin{array}{l}\text { A review of the field of microbial biosynthetic } \\
\text { methods for GAG synthesis, with a focus on } \\
\text { supporting biomedical use of GAGs, is } \\
\text { provided in this work. It identifies microbial } \\
\text { species that naturally synthesize unsulfated } \\
\text { GAGs, identifies key enzymes involved in the } \\
\text { biosynthetic process, describes methods for } \\
\text { microbial fermentation for mass producaiton of } \\
\text { the GAGs, and finally, discusses post- } \\
\text { translational modifications. }\end{array}$ \\
\hline
\end{tabular}

Wiley Interdiscip Rev Nanomed Nanobiotechnol. Author manuscript; available in PMC 2014 July 01. 
Table 2

Reviews of GAGs and Their Use as Surface Coatings and as Components of Materials

\begin{tabular}{|c|c|}
\hline Reference & Content \\
\hline $\begin{array}{l}\text { Jia and Kiick, Macromolecular Biosciences, } \\
\text { 2009, 9:140-156. }\end{array}$ & $\begin{array}{l}\text { This review covers biohybrid hydrogels } \\
\text { including protein-synthetic and GAG-synthetic } \\
\text { hybrids. The GAG-hybrid gels discussed } \\
\text { include hyaluronic acid-synthetic and heparin- } \\
\text { synthetic gels and their uses in tissue } \\
\text { engineering and biopolymer drug delivery. }\end{array}$ \\
\hline $\begin{array}{l}\text { Baldwin and Kiick, Peptide Science, } 2010 \text {, } \\
94: 128-140.55\end{array}$ & $\begin{array}{l}\text { This review discusses the conjugation of } \\
\text { polysaccharides to synthetic polymers and their } \\
\text { impact on in vivo applications. Mammalian, as } \\
\text { well as microbial and plant polysaccharides are } \\
\text { discussed. }\end{array}$ \\
\hline $\begin{array}{l}\text { Hudalla and Murphy, Advanced Functional } \\
\text { Materials, 2011, 21:1754-1768. }\end{array}$ & $\begin{array}{l}\text { This review focuses on utilization of GAGs, } \\
\text { polyglyco-mimetics and polyelectrolytes for } \\
\text { growth factor delivery. While inclusive of } \\
\text { materials preloaded with growth factors, this } \\
\text { work describes novel methods to sequester, } \\
\text { present and release growth factors secreted by } \\
\text { cells cultured on or near the materials. }\end{array}$ \\
\hline
\end{tabular}

Wiley Interdiscip Rev Nanomed Nanobiotechnol. Author manuscript; available in PMC 2014 July 01. 
Table 3

Reviews Examining Blends of GAGs and Other Biopolymers

\begin{tabular}{ll}
\hline Reference & Content \\
\hline $\begin{array}{l}\text { Ferdous and Grande-Allen, Tissue } \\
\text { Engineering, 2007, 13:1893-1904. }{ }^{57}\end{array}$ & $\begin{array}{l}\text { This review addresses various methods of } \\
\text { integrating proteoglycans within engineered } \\
\text { tissues and examines the design of tissue- } \\
\text { engineered disease models utilizing } \\
\text { proteoglycan biology. }\end{array}$ \\
\hline $\begin{array}{l}\text { Yannas, et al., Philosophical Transactions. } \\
\text { Series A, Mathematical, Physical, and } \\
\text { Engineering Sciences, 2010, 368:2123-2139. }{ }^{58}\end{array}$ & $\begin{array}{l}\text { This review describes detailed methodologies } \\
\text { for both the synthesis and characterization of } \\
\text { collagen-glycosaminoglycan scaffolds. }\end{array}$ \\
\hline
\end{tabular}

Wiley Interdiscip Rev Nanomed Nanobiotechnol. Author manuscript; available in PMC 2014 July 01. 